\title{
Diseño y formulación de mezclas de combustible tipo Glow con desarrollo metodológico basado en ANOVA
}

Design and formulation of Glow type fuel blends with methodological development based on ANOVA

Création et réalisation de mélanges de combustibles de tipe glow grâce aux améliorations méthodologiques de I'ANOVA

\section{Desenho e formulação de misturas de combustível tipo glow com desenvolvimento metodológico baseado em ANOVA}

\section{Gerardo Avendaño Prieto * Víctor Orlando Zambrano G. ** José Alejandro Martínez***}

Fecha de recepción: 22 de abril de 2015

Fecha de aprobación: 10 de junio de 2015

Pp.7-28

* Postdoctor en Manufacturing Engineering, Penn State University. Métodos Estadísticos Avanzados, Universidad Politécnica de Valencia. Profesor Titular, Universidad EAN.

** Magíster(c) en Ingeniería de Procesos, Universidad EAN. Ingeniero Mecánico, Universidad Nacional de Colombia.

*** Magíster en Ingeniería Ambiental e Ingeniero Químico, Universidad Nacional de Colombia. Director de la Maestría en Ingeniería de Procesos de la Universidad EAN. Docente asociado e investigador, Universidad EAN. 


\section{RESUMEN}

El presente artículo, trata sobre una investigación para el diseño y formulación de mezclas de combustible tipo Glow para motores de aeromodelos, que con un planteamiento metodológico permite seleccionar la mejor mezcla combustible para un motor de dos tiempos particular, en condiciones particulares, y que adicionalmente acepta plantear como objetivo, el de socializar la metodología experimental y estadística conocida como diseño de experimentos para la generación y el tratamiento de los datos derivados de un experimento con su previa formulación, permitiendo a los interesados, abordar investigaciones en motores y plazas alternativas.

\section{Palabras Clave}

Motor Glow, mezcla combustible Glow, gasolina Glow, diseño de experimentos, aeromodelismo, metodología para investigación, Nitrometano $100 \%$, ANOVA.

\section{Abstract}

This article deals with an investigation directed at designing and formulating Glow type fuel mixtures for model aircraft engines which, with a methodological approach, enables the selection of the best fuel mixture for two-stroke engines under particular conditions, and that additionally accepts to aim at socializing the experimental and statistical methodology known as design of experiments for the generation and treatment of data derived from an experiment with previous formulation, allowing interested parties to address research on alternative engines and spaces.

\section{KEYWORDS}

Motor Glow, glow fuel, design of experiments, research methodology, nitromethane, nitromethane $100 \%$, ANOVA. 


\section{RÉSUMÉ}

Cet article présente une recherche réalisée pour la création et réalisation de mélanges de combustibles de type Glow pour moteurs d'appareils volants qui grâce aux exposés méthodologiques peuvent sélectionner le meilleur mélange de combustible pour un moteur deux temps dans des conditions de fonctionnement spécifiques. Il s'agit de plus de partager la méthodologie expérimentale et les statistiques connues pour la conceptualisation d'expériences pour la création et le traitement des données dérivées $d^{\prime}$ expériences permettant aux intéressés d'aborder des investigations sur les moteurs et zones alternatives.

\section{MOTS CLEFS}

Moteur Glow, Combustible Glow, Création d'Expériences, Méthodologie de recherche, Nitrométhane, Nitrométhane 100\%, ANOVA.

\section{Resumo}

O presente artigo, trata sobre uma investigação para o desenho e formulação de misturas de combustíveis tipo Glow para motores de aeromodelos, que com uma abordagem metodológica, permite selecionar a melhor mistura combustível para um motor de dois tempos particular, em condições particulares, e que adicionalmente aceita apresentar como objetivo, o de socializar a metodologia experimental e estatística conhecida como desenho de experimentos para a geração e o tratamento dos dados derivados de um experimento com sua prévia formulação, permitindo aos interessados, abordar pesquisas científicas em motores alternativos.

\section{Palavras-chave}

Tempo de secado, verniz, catalizador, diluente, Anova, fatores, níveis, variável de resposta, produtividade, eficiência. 


\section{Introducción}

- n esta investigación se aborda el desarrollo de un tipo especial y poco común, dado el reducido círculo en el que se requiere de una mezcla combustible tipo Glow para los motores de dos tiempos, cuya preparación adolece de experiencias sólidas documentadas y de información estructurada; por esta razón, se aborda con el objetivo de caracterizar una mezcla combustible que, a la altura de Bogotá D. C., determine el mejor desempeño del motor seleccionado, el Super Tigre GS-40, que se observará mediante la experimentación directa y la medición de la temperatura en la culata -cabeza del motor o tapa del cilindro- y la velocidad de giro de la hélice, como indicadores de generación de energía a partir del combustible y su transformación.

El alcance de la investigación corresponde con la definición de una mezcla combustible de tipo Glow, es decir, para motores tipo Glow, que permita obtener el mejor desempeño en el motor seleccionado en las condiciones de altura de la ciudad de Bogotá D. C.

Dado que la presente investigación sustenta sus resultados en el diseño de experimentos y que tanto las limitaciones económicas para la formulación y preparación de las mezclas como la disponibilidad del motor, dan tiempo para la experimentación, y además, considerando que los espacios requeridos no permitieron la realización de réplicas a los ensayos, la investigación y sus resultados se reportarán únicamente sobre los 16 ensayos determinados por el plan $2^{k}$ establecido.

Derivado de lo expuesto en el cuerpo de este artículo científico con su alcance y limitaciones, quedan establecidas las bases de una metodología formal, pero secuencial y sencilla 
para los modelistas, de tal manera que les permita conducir investigaciones soportadas en el modelo propuesto que puedan ser llevadas a cabo con motores diferentes, en plazas alternativas. Esta metodología desarrolla formatos y cuadros en MS-Excel, basados en los planes factoriales con factores a dos niveles, planes $2^{\mathrm{k}}$.

La experimentación registrada se llevó a cabo en un motor Super Tigre GS-40, el cual se preparó mecánicamente; así mismo, se desarrolló el montaje tipo laboratorio para los respectivos ensayos. Una vez dispuesto lo anterior, se diseñó la tabla modelo en la cual se registró la propuesta de factores con sus niveles llevados a valores y relaciones que determinaron la caracterización de las respectivas mezclas.

El presente artículo se ha estructurado en siete acápites que permiten, de manera secuencial, exponer la investigación, su estructura y resultados, a saber: introducción, marco teórico, método, resultados, conclusiones, bibligrafía y anexo.

\section{Marco teórico}

uando en el círculo de modelistas se indaga sobre el combustible Glow, se cae inmediatamente en el terreno de lo desconocido y lo anecdótico, pues no existe documentación técnica para el diseño y la preparación de diferentes tipos de combustible, lo que no permite mejorar el desempeño de los motores en las diferentes plazas en las que se operan, por recreación, deporte o com-petencia, pues en general se manejan mezclas estándar definidas por fabricantes como Omega, Tower Power, Byron Fuel, Cool Power, Voldcat, Model Technics, etc. 
Dado que los componentes del combustible Glow son productos químicos de cuidadoso manejo, no resulta sencilla su adecuada y segura mezcla, aunque se encuentran entusiastas que han abordado el tema con muy buenos resultados.

Los logros alcanzados y el entendimiento del desempeño de los motores Glow a partir del diseño y modificación de los combustibles, se transmite voz a voz, por lo cual no se maneja alguna estructuración de la información y no se difunde de manera fidedigna en la mayoría de los casos. No resulta difícil encontrar una gran cantidad de artículos informales, en páginas web $y$ en blogs de aficionados al tema de los combustibles Glow, lo cual crea confusión ante la inmensa variedad de fórmulas que prometen ser la mejor solución al desempeño de los motores.

Existe por tanto, un gran vacío metodológico para la evaluación de mezclas de combustible, en términos de cuáles son los componentes más adecuados y sus proporciones, y finalmente, qué se espera del desempeño de los motores en términos de temperatura y velocidad de la hélice.

La revisión de la información disponible y de las fuentes reconocidas por los modelistas, determina que los compuestos químicos comunes a las diferentes mezclas son Metanol 100\% $\left(\mathrm{CH}_{4} \mathrm{O}\right)$, Nitrometano $100 \%\left(\mathrm{CH}_{3} \mathrm{NO}_{2}\right)$, Acetato de Amilo $\left(\mathrm{C}_{7} \mathrm{H}_{14} \mathrm{O}_{2}\right)$, Aceite de Ricino (desgomado) y Aceite Mineral o Sintético.

La presente investigación se realiza en concordancia con los objetivos propuestos pero sin perder de vista la actualidad tecnológica de los modelistas, que los ha llevado a abandonar los motores Glow ya calificados en principio de obsolescencia, y a adoptar los motores eléctricos de nueva generación, cuyas prestaciones les exceden en mucho, pues son relativamente económicos, entregan más del $95 \%$ de la energía en potencia motriz, tienen muy alta relación potencia Vs. peso y tamaño, son silenciosos y muy fácilmente controlables por ordenador. 
La metodología utilizada para el análisis de datos dentro del diseño de experimentos, es el tratamiento ANOVA, el cual facilita la realización de un tratamiento integral de la colección de datos adquiridos, para convertirlos en información útil que permita el alcance del objetivo técnico.

\section{Método}

I método utilizado en la presente investigación, se centra en el diseño de experimentos con experimentación directa y con profusa adquisición de datos para el posterior análisis de estos, con métodos estadísticos caso conocido como experimento factorial $2^{k}$, en el que se estudia el efecto sobre una variable de respuesta de $k$ factores con dos niveles preestablecidos.

La definición de la variable de respuesta y de los niveles para los $k$ factores, se realiza con base en las condiciones técnicas de conocimiento del motor y en el conocimiento empírico difundido en el mercado del aeromodelismo sobre las mezclas combustible.

La estructura y definición del experimento se tiene de la siguiente manera: 


\subsection{Definición del problema y establecimiento del objetivo}

En la actualidad no se dispone de un conocimiento estructurado y documentado sobre alternativas de mezclas combustible tipo Glow, por lo que resulta difícil obtener desempeños mejorados en los motores de tal tipo, dependiendo de las plazas en las que se operan, lo que se ha convertido en un problema no formulado y por lo tanto no estudiado. Lo anterior se constituye en el objetivo principal, por lo que se determina en este experimento, la formulación y selección de una mezcla combustible tipo Glow, que permitirá encontrar el mejor desempeño del motor seleccionado en las condiciones de altitud de la ciudad de Bogotá D. C., que en conjunto con el planteamiento realizado de la metodología de análisis, generará conocimiento metodológicamente estructurado.

\subsection{Identificación de la variable de respuesta}

La temperatura es un indicador de la actividad energética en las máquinas de combustión interna, a través de la cual se manifiesta la energía química del combustible en su transformación hacia la producción de trabajo mecánico. Resulta entonces claro que, una mayor temperatura detectada indica más poder energético del combustible, lo cual redunda en mayor potencia mecánica disponible; lo anterior, en el entendido de que el motor dispone de la correcta lubricación y refrigeración, de lo contrario, gran parte de la temperatura sería generada por rozamientos mecánicos y falta de refrigeración.

El motor usado en la experimentación, está equipado con una hélice denominada $10 \times 6$ de dos palas, la que le consume la 
energía mecánica disponible, medición que de manera directa se tiene en las revoluciones por minuto a plena velocidad. Aunque la velocidad de la hélice es una manifestación clara del funcionamiento del motor, se determina que será la temperatura la variable de respuesta, dado que, en buenas condiciones del motor, es una clara evidencia de la transformación de la energía química del combustible.

\subsection{Selección de los factores}

Los factores seleccionados son los componentes minoritarios reconocidos en la mayoría de las mezclas, a saber, Nitrometano $100 \%\left(\mathrm{CH}_{3} \mathrm{NO}_{2}\right)$, Acetato de Amilo $\left(\mathrm{C}_{7} \mathrm{H}_{14} \mathrm{O}_{2}\right)$, Aceite de Ricino (desgomado) y Aceite Sintético.

Esto determina un plan factorial $2^{k}$, con cuatro factores.

\subsection{Definición de los niveles -variantes cuantitativas-}

La definición de los niveles de cada factor, se basa en las proporciones que el saber empírico de los modelistas y fabricantes de combustibles tienen por cierto. Con un ajuste conveniente para cada uno en sus valores mínimos y máximos, estos niveles serán:

- Nitrometano $100 \%\left(\mathrm{CH}_{3} \mathrm{NO}_{2}\right)$ : mínimo $5 \%$, máximo $15 \%$

- Aceite Sintético: mínimo $15 \%$, máximo $20 \%$.

- Aceite de Ricino (desgomado): mínimo 1\%, máximo 3\%. 
- Acetato de Amilo $\left(\mathrm{C}_{7} \mathrm{H}_{14} \mathrm{O}_{2}\right)$ : mínimo 0,0\%, máximo 0,4\%.

El Metanol $100 \%\left(\mathrm{CH}_{4} \mathrm{O}\right)$, siendo el vehículo de la mezcla combustible, se formulará en el nivel que corresponda a c.s.p. $100 \%$, es decir, cantidad suficiente para $100 \%$ en volumen.

\subsection{Definición de cada prueba individual}

Una vez definido el nivel de cada factor, se determina la realización de $100 \mathrm{ml}$ de cada mezcla, de tal manera que siendo el Metanol el vehículo de estas, se proporcione como c.s.p. $100 \mathrm{ml}$.

Cada mezcla, en cantidad suficiente en el tanque de combustible del montaje del motor, permite que se realice cada prueba durante el tiempo debido para carburar la mezcla y obtener los datos de temperatura y velocidad.

Una información que resulta importante desde el punto de vista operativo, es la facilidad con que cada mezcla se deja carburar en el motor durante la prueba, pues esto permite identificar, conceptualmente aquellas mezclas que presentan características superiores para la combustión.

\subsection{Definición del número de pruebas a realizar}

Un plan factorial $2^{k}$, con cuatro $(k=4)$ factores, determina la realización de 16 pruebas, es decir, 16 diferentes mezclas combustible de prueba. 


\subsection{Definición del tratamiento a utilizar en cada una de ellas}

El tratamiento corresponde a la formulación propiamente dicha, la cual en conjunto con el planteamiento general, la definición de los factores y sus niveles, se registran más adelante (Figura 1). El modelo mostrado se desarrolló para la presente investigación en MS-Excel, automatizado hasta el punto requerido de tal manera que la certeza en la formulación de las diferentes mezclas es del ciento por ciento, una vez comprobadas todas las fórmulas de la hoja.

Figura 1. Diseño del experimento.

\begin{tabular}{|c|c|c|c|c|c|c|c|c|c|}
\hline \multirow{5}{*}{\multicolumn{5}{|c|}{$\begin{array}{l}\text { DISENO DE EXPERIMENTOS PARA } \\
\text { COMBUSTIBLE GLOW 2T }\end{array}$}} & \multicolumn{5}{|c|}{ PLANTEAMIENTO } \\
\hline & & & & & \multirow{2}{*}{\multicolumn{2}{|c|}{ Nitrometano $\mathrm{CH}_{3} \mathrm{NO}_{2}$}} & F1- & $5,0 \%$ & \multirow{4}{*}{$\begin{array}{c}\text { Tenperaturara } \\
\text { en ta buja. } \\
2000^{\circ} \mathrm{C} \\
\pm 15^{\circ} \mathrm{C}\end{array}$} \\
\hline & & & & & & & \multicolumn{2}{|c|}{$15,0 \%$} & \\
\hline & & & & & \multicolumn{2}{|c|}{ Aceite Sintético } & $\mathrm{F} 2$ & $20,0 \%$ & \\
\hline \multirow{3}{*}{\multicolumn{3}{|c|}{ VOLUMEN DE REFERENCI }} & & & \multicolumn{2}{|c|}{ Aceite Ricino } & \multicolumn{2}{|c|}{$1,0 \%$} & \\
\hline & & & \multirow{2}{*}{\multicolumn{2}{|c|}{$100 \mathrm{cc}$}} & \multirow{2}{*}{\multicolumn{2}{|c|}{$\begin{array}{l}\text { Acetato de Amilo } \\
\mathrm{C}_{7} \mathrm{H}_{14} \mathrm{O}_{2}\end{array}$}} & \multirow{2}{*}{\multicolumn{2}{|c|}{$\begin{array}{ll} & 0,0 \% \\
& \\
\end{array}+, 4 \%$}} & \multirow{2}{*}{$\begin{array}{c}3 \min \\
\pm 1 \min \end{array}$} \\
\hline & & & & & & & & & \\
\hline \multicolumn{5}{|c|}{ TRATAMIENTOS FACTORES \& NIVELES } & \multicolumn{5}{|c|}{ FORMULACIÓN DE MEZCLAS } \\
\hline Tratamiento & $\begin{array}{c}\mathrm{F} 1 \\
\text { Nitrometano } \\
\mathrm{CH}_{3} \mathrm{NO}_{2}\end{array}$ & $\begin{array}{c}\text { F2 } \\
\text { Aceite Sintético }\end{array}$ & $\begin{array}{c}\text { F3 } \\
\text { Accite de Ricino }\end{array}$ & $\begin{array}{l}\text { F4 } \\
\text { Acetato de } \\
\text { Amilo } \\
\mathrm{C}_{7} \mathrm{H}_{\mathrm{H}_{4} \mathrm{O}_{2}}\end{array}$ & $\begin{array}{c}\mathbf{F 1} \\
\text { Nitrometano } \\
\mathrm{CH}_{3} \mathrm{NO}_{2} \\
\end{array}$ & $\begin{array}{c}\text { F2 } \\
\text { Aceite } \\
\text { Sintético }\end{array}$ & $\begin{array}{c}\text { F3 } \\
\text { Aceite de } \\
\text { Ricino }\end{array}$ & $\begin{array}{l}\mathrm{F} 4 \\
\text { Acetato de } \\
\text { Amilo } \\
\mathrm{C}_{7} \mathrm{H}_{14} \mathrm{O}_{2}\end{array}$ & $\begin{array}{c}\text { c.s.p. } 100 \% \\
\text { Metanol } \\
\mathrm{CH}_{4} \mathrm{O}\end{array}$ \\
\hline E01 & $5,0 \%$ & $15,0 \%$ & $1,0 \%$ & $0,0 \%$ & 5,00 & 15,00 & 1,00 & 0,00 & 79,00 \\
\hline E02 & $15,0 \%$ & $15,0 \%$ & $1,0 \%$ & $0,0 \%$ & 15,00 & 15,00 & 1,00 & 0,00 & 69,00 \\
\hline E03 & $5,0 \%$ & $20,0 \%$ & $1,0 \%$ & $0,0 \%$ & 5,00 & 20,00 & 1,00 & 0,00 & 74,00 \\
\hline E04 & $15,0 \%$ & $20,0 \%$ & $1,0 \%$ & $0,0 \%$ & 15,00 & 20,00 & 1,00 & 0,00 & 64,00 \\
\hline E05 & $5,0 \%$ & $15,0 \%$ & $3,0 \%$ & $0,0 \%$ & 5,00 & 15,00 & 3,00 & 0,00 & 77,00 \\
\hline E06 & $15,0 \%$ & $15,0 \%$ & $3,0 \%$ & $0,0 \%$ & 15,00 & 15,00 & 3,00 & 0,00 & 67,00 \\
\hline E07 & $5,0 \%$ & $20,0 \%$ & $3,0 \%$ & $0,0 \%$ & 5,00 & 20,00 & 3,00 & 0,00 & 72,00 \\
\hline E08 & $15,0 \%$ & $20,0 \%$ & $3,0 \%$ & $0,0 \%$ & 15,00 & 20,00 & 3,00 & 0,00 & 62,00 \\
\hline E09 & $5,0 \%$ & $15,0 \%$ & $1,0 \%$ & $0,4 \%$ & 5,00 & 15,00 & 1,00 & 0,40 & 78,60 \\
\hline E10 & $15,0 \%$ & $15,0 \%$ & $1,0 \%$ & $0,4 \%$ & 15,00 & 15,00 & 1,00 & 0,40 & 68,60 \\
\hline E11 & $5,0 \%$ & $20,0 \%$ & $1,0 \%$ & $0,4 \%$ & 5,00 & 20,00 & 1,00 & 0,40 & 73,60 \\
\hline E12 & $15,0 \%$ & $20,0 \%$ & $1,0 \%$ & $0,4 \%$ & 15,00 & 20,00 & 1,00 & 0,40 & 63,60 \\
\hline E13 & $5,0 \%$ & $15,0 \%$ & $3,0 \%$ & $0,4 \%$ & 5,00 & 15,00 & 3,00 & 0,40 & 76,60 \\
\hline E14 & $15,0 \%$ & $15,0 \%$ & $3,0 \%$ & $0,4 \%$ & 15,00 & 15,00 & 3,00 & 0,40 & 66,60 \\
\hline E15 & $5,0 \%$ & $20,0 \%$ & $3,0 \%$ & $0,4 \%$ & 5,00 & 20,00 & 3,00 & 0,40 & 71,60 \\
\hline E16 & $15,0 \%$ & $20,0 \%$ & $3,0 \%$ & $0,4 \%$ & 15,00 & 20,00 & 3,00 & 0,40 & 61,60 \\
\hline & Disp & (1) & the th & $-x^{2}$ & 160,0 & 280,0 & 32,0 & 3,2 & 1124,8 \\
\hline
\end{tabular}

Fuente. Elaboración propia de los autores, a partir de MS-Excel, (s.f). 


\subsection{Organización de todo el trabajo experimental}

La organización del trabajo experimental se describe a continuación:

- Realización de las mezclas de combustible bajo estándar metodológico y de calidad en el laboratorio de química de la Universidad EAN ${ }^{1}$. Se observa la disposición para la mezcla de las diferentes fórmulas (Figura 2). Los productos químicos se transportaron en los contenedores de suministro; las mezclas preparadas se almacenaron en frascos de $100 \mathrm{ml}$, graduados y autoclavables, con cuello y rosca según las normas DIN 13361 y DIN 168; los frascos fueron debidamente rotulados de acuerdo con la codificación del diseño de experimento para cada mezcla contenida.

Figura 2. Preparación de mezclas.

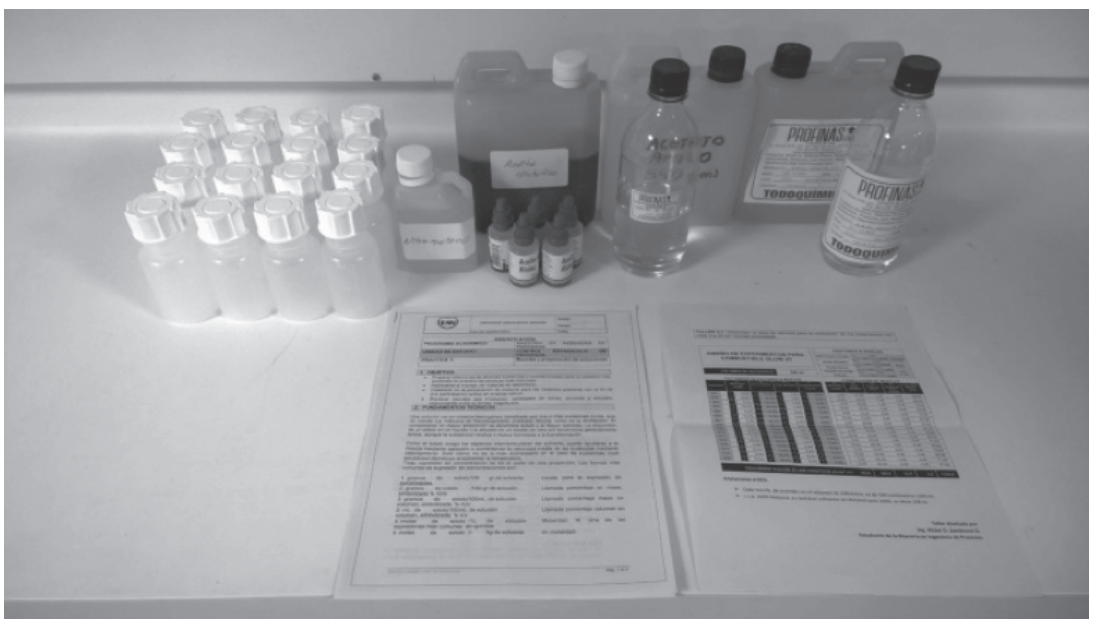

Fuente. Elaboración propia de los autores, (s.f).

1 En anexo el documento metodológico original. 
- Para la experimentación directa, se debió disponer de los siguientes elementos:

» Montaje realizado en madera laminada, armado con dos placas atornilladas, para garantizar la resistencia a los ensayos con el motor de $2 \mathrm{HP}$ de potencia. Utilización de manguera de silicona para combustible (Figura 3).

Figura 3. Montaje del motor con capacidad para 2 HP.

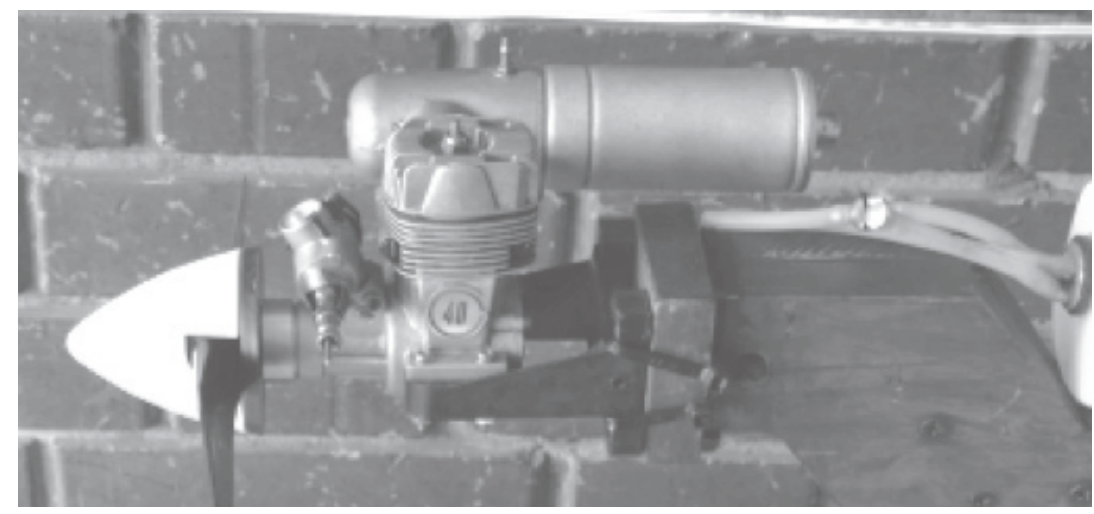

Fuente. Elaboración propia de los autores, (s.f).

» Termómetro infrarrojo sin contacto, digital con rango de medición $-60^{\circ} \mathrm{C} \pm 1,5^{\circ} \mathrm{C}$ a $550^{\circ} \mathrm{C} \pm 1,5^{\circ} \mathrm{C}$, tiempo de respuesta de $1 \mathrm{~s}$ y emisividad para el material de motor de 0,30 (Figura 4). 
Figura 4. Termómetro infrarrojo sin contacto, digital.

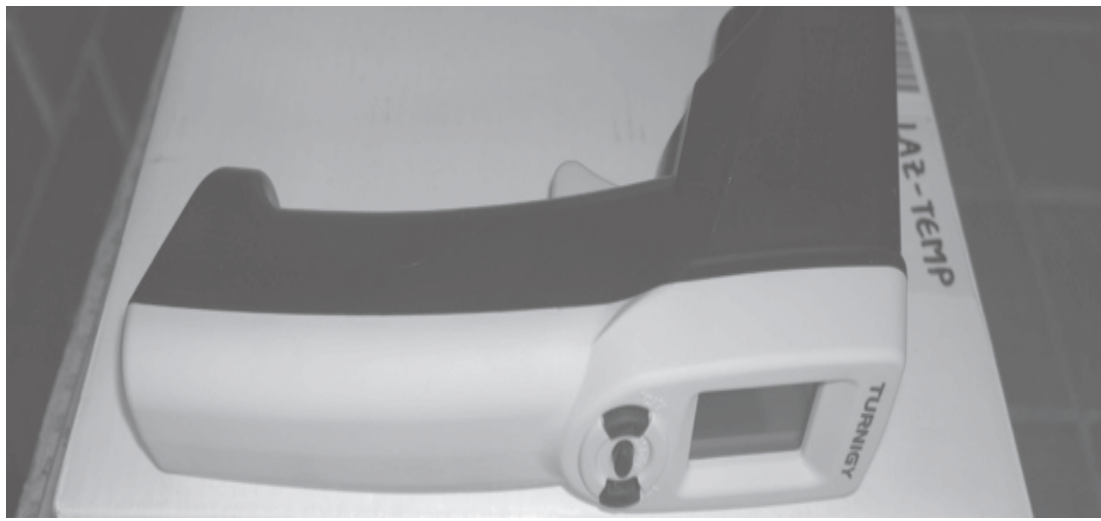

Fuente. Elaboración propia de los autores, (s.f).

» Tacómetro digital con capacidad para medir en hélices con dos a nueve palas y velocidades de giro hasta de 99999 rpm (Figura 5).

Figura 5. Tacómetro digital para medir hélices.

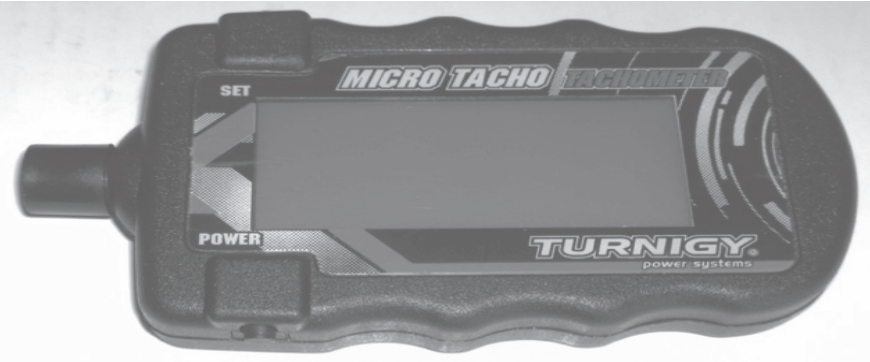

Fuente. Elaboración propia de los autores, (s.f). 
"Los tratamientos o ensayos se realizaron en campo abierto en la ciudad de Bogotá D. C., en horas de la mañana, con una temperatura ambiental de $18^{\circ} \mathrm{C}$ y una humedad relativa del $55 \%$, medición realizada localmente en el entorno del ensayo (Figura 6).

Figura 6. Realización de tratamientos.

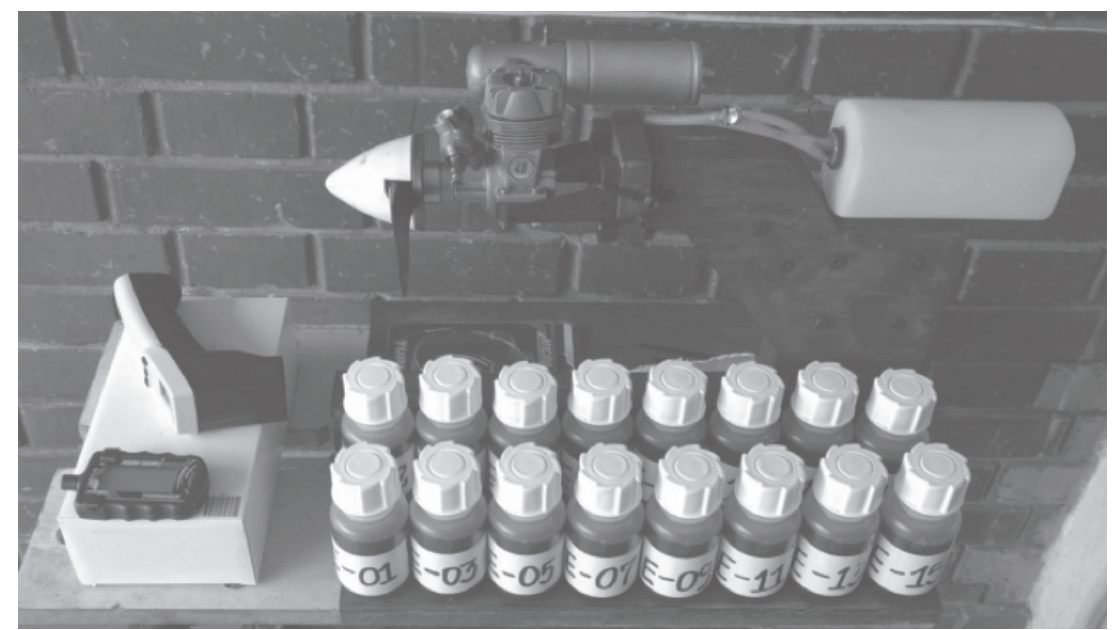

Fuente. Elaboración propia de los autores, (s.f).

»Para la toma de datos, se diseñó el formato que se muestra a continuación (Figura 7), el cual indica los registros requeridos para asegurar la calidad de los tratamientos y la máxima rigurosidad en el manejo de la información. Este permite el registro de los resultados de cada uno de los 16 tratamientos. 
Figura 7. Realización de la toma de datos

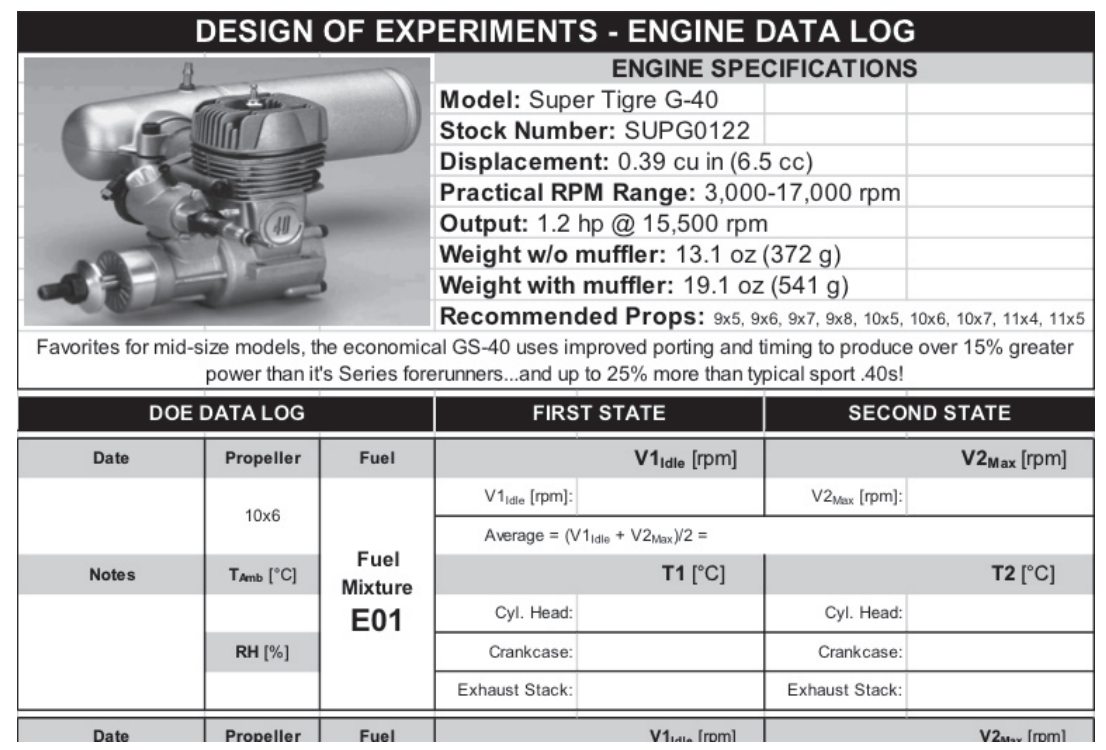

Fuente. Elaboración propia de los autores, a partir de MS-Excel, (s.f).

\subsection{Realización del análisis estadístico de los resultados}

Dado que la base del análisis estadístico corresponde con los planes factoriales, con factores a dos niveles, planes $2^{k}$, lo que sigue es la correspondiente metodología acompañada de cuadros diseñados para tal fin, derivados del modelo matemático en MS-Excel debidamente formulado y orientado a facilitar el entendimiento de la metodología general.

Se observa (Figura 8) en las últimas columnas, el resultado de temperatura del motor y velocidad de hélice para cada tratamiento, tomadas en la culata de este y en la hélice misma respectivamente, durante la experimentación directa. 
Figura 8. Tabla de signos para el análisis de las interacciones y la realización de los respectivos promedios, según la metodología ANOVA

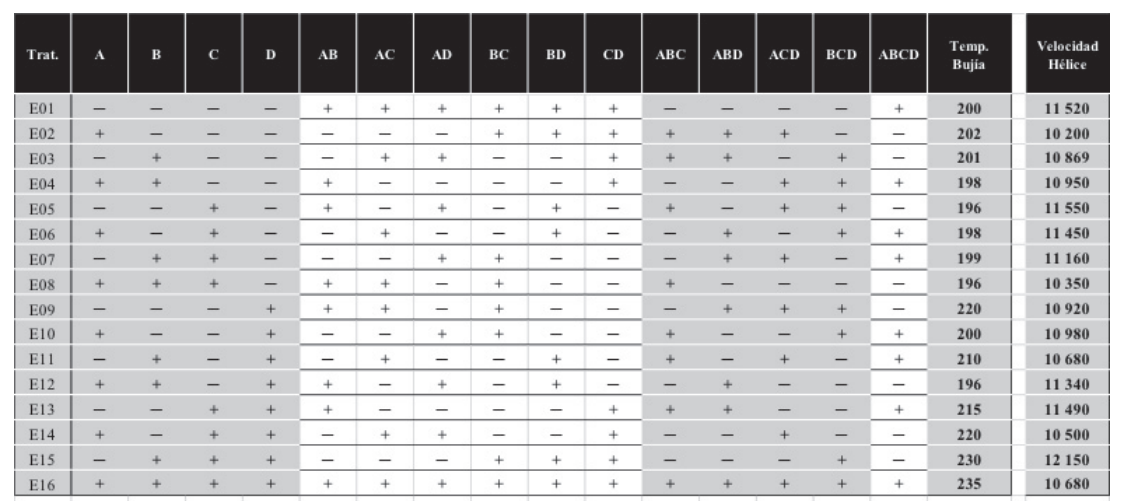

\begin{tabular}{|l|l|l|l|l|l|l|l|l|l|l|l|l|l|l|l|}
$-3,25$ & 1,75 & 7,75 & 17,00 & $-0,50$ & 5,50 & $-2,75$ & 6,00 & 2,25 & 10,75 & $-0,75$ & 2,00 & 5,50 & 5,00 & $-0,75$ & 207 \\
\hline
\end{tabular}

11049

Fuente. Elaboración propia de los autores, a partir de MS-Excel, (s.f).

Una vez realizada la tabla de signos, se procedió con la elaboración de la primera de dos tablas de resultados ANOVA, de tal manera que se generan en concordancia con la formulación indicada a continuación (Figura 9).

Figura 9. ANOVA Summary Table

\begin{tabular}{|c|c|c|c|c|c|}
\hline Variation & S.C. & g.l. & C.M. & F-Ratio & F-Table \\
\hline Total & $\sum_{i=1}^{n}\left(x_{i}-\overline{\bar{x}}\right)^{2}$ & $n-1$ & $\frac{1}{n-1} \sum_{i=1}^{n}\left(x_{i}-\overline{\bar{x}}\right)^{2}$ & & \\
\hline Treatment & $\sum_{i=1}^{n} n_{i}\left(x_{i}-\overline{\bar{x}}\right)^{2}$ & $k-1$ & $\frac{1}{k-1} \sum_{i=1}^{n} n_{i}\left(x_{i}-\overline{\bar{x}}\right)^{2}$ & $\frac{C . M_{\text {Treatment }}}{C . M_{\text {Residual }}}$ & \\
\hline Residual & $S . C_{\text {Total }}-S . C \cdot_{\text {Treatment }}$ & $n-k$ & $\frac{S . C_{\cdot_{\text {Total }}}-S . C_{\cdot_{\text {Treatment }}}}{n-k}$ & & \\
\hline Reply & \multicolumn{5}{|c|}{ Statistically significant effects are ... } \\
\hline $\begin{array}{r}\text { Expected } \\
\text { Average }\end{array}$ & \multicolumn{5}{|c|}{$x+\frac{1}{2} \sum$ Statistically Significant Effects } \\
\hline
\end{tabular}

Fuente. Elaboración propia de los autores, a partir de MS-Word, (s.f). 
De acuerdo con lo anterior, se registran todos los factores y todas las interacciones para un total de dieciséis (Figura 10).

Figura 10. Tabla de Resultados ANOVA (1/2).

\begin{tabular}{|c|r|r|r|l|l|}
\hline Efecto & $\begin{array}{c}\text { Suma } \\
\text { cuadrados } \\
\text { S.C. }\end{array}$ & $\begin{array}{c}\text { Grados de } \\
\text { libertad } \\
\text { g.l. }\end{array}$ & $\begin{array}{c}\text { Cuadrado } \\
\text { medio } \\
\text { S.C./g.I. }\end{array}$ & F-Ratio & F-Tablas \\
\hline Total & $2.471,00$ & 15 & 164,73 & & \\
\hline A & 42,25 & 1 & 42,25 & & \\
\hline B & 12,25 & 1 & 12,25 & & \\
\hline C & 240,25 & 1 & 240,25 & & \\
\hline D & $1.156,00$ & 1 & $1.156,00$ & & \\
\hline AB & 1,00 & 1 & 1,00 & & \\
\hline$A C$ & 121,00 & 1 & 121,00 & & \\
\hline$A D$ & 30,25 & 1 & 30,25 & & \\
\hline BC & 144,00 & 1 & 144,00 & & \\
\hline BD & 20,25 & 1 & 20,25 & & \\
\hline CD & 462,25 & 1 & 462,25 & & \\
\hline ABC & 2,25 & 1 & 2,25 & & \\
\hline ABD & 16,00 & 1 & 16,00 & & \\
\hline ACD & 121,00 & 1 & 121,00 & & \\
\hline BCD & 100,00 & 1 & 100,00 & & \\
\hline ABCD & 2,25 & 1 & 2,25 & & \\
\hline Residual & & & & & \\
\hline
\end{tabular}

Fuente. Elaboración propia de los autores, a partir de MS-Excel, (s.f).

A continuación, se identifican los efectos estadísticamente significativos (Figura 11). 
Figura 11. Tabla de Resultados ANOVA (2/2).

\begin{tabular}{|r|r|r|r|r|r|}
\hline Efecto & $\begin{array}{c}\text { Suma } \\
\text { Cuadrados } \\
\text { S.C. }\end{array}$ & $\begin{array}{c}\text { Grados de } \\
\text { Libertad } \\
\text { g.l. }\end{array}$ & $\begin{array}{c}\text { Cuadrado } \\
\text { Medio } \\
\text { S.C./g.l. }\end{array}$ & F-Ratio & F-Tablas \\
\hline Total & 2471,00 & 15 & 164,73 & & \\
\hline A & 42,25 & 1 & 42,25 & 1,834 & 5,320 \\
\hline C & 240,25 & 1 & 240,25 & 10,431 & 5,320 \\
\hline D & 1156,00 & 1 & 1156,00 & 50,193 & 5,320 \\
\hline AC & 121,00 & 1 & 121,00 & 5,254 & 5,320 \\
\hline BC & 144,00 & 1 & 144,00 & 6,252 & 5,320 \\
\hline CD & 462,25 & 1 & 462,25 & 20,071 & 5,320 \\
\hline ACD & 121,00 & 1 & 121,00 & 5,254 & 5,320 \\
\hline Residual & 184,25 & 8 & 23,03 & & \\
\hline CRITERIO... Si F-ratio es $\geq$ que F-tablas el efecto es estadisticamente significativo. \\
\hline REGLA... Los Grados de Libertad Residual (g. . $_{\text {Residual }}$ ) deben ser mayores o iguales a cuatro $(\geq 4)$ \\
\hline
\end{tabular}

Fuente. Elaboración propia de los autores, a partir de MS-Excel, (s.f).

Finalmente, se tiene que la Media Prevista (M.P.) que se determina como:

$$
\text { M.P. }=\bar{X}+\frac{1}{2} \sum \text { Efectos Estadísticamente Significativos }=1,5 s
$$

Tendrá como resultado, para la temperatura en la culata -cabeza del motor o tapa del cilindro-:

$$
M . P .=207,3+\frac{1}{2}(7,75+17,00+6,00+10,75)=228,05
$$

\section{Resultados}

os resultados obtenidos y registrados recientemente, nos muestran estadísticamente los efectos $C, D, B C, C D$. Esto quiere decir que el Acetato de Amilo $\left(\mathrm{C}_{7} \mathrm{H}_{14} \mathrm{O}_{2}\right)$, Aceite de Ricino (desgomado) y Aceite Mineral o Sintético, que son la base de la mayoría de mezclas en el mercado, resultan ser los componentes significativos en las mezclas analizadas. 
El componente de mayor poder detonante, Nitrometano $100 \%\left(\mathrm{CH}_{3} \mathrm{NO}_{2}\right)$, en sus niveles $5 \%$ y $15 \%$, no resulta estadísticamente significativo, pero se debe registrar que la mezcla E15 produjo una de las mayores temperaturas y la mayor velocidad de hélice, lo cual confirma su capacidad detonante; de manera similar se pueden observar las mezclas E13, E14, E15 y E16, que por su contenido de Nitrometano $100 \%\left(\mathrm{CH}_{3} \mathrm{NO}_{2}\right)$, se obtuvo en conjunto el mejor desempeño del motor.

\section{Conclusiones}

I tener el desarrollo del experimento basado en un plan $2^{k}$, sin réplicas, está claro que el error subsecuente puede resultar significativo, para lo cual un experimento ulterior deberá ser planteado con por lo menos, dos réplicas.

Sin embargo, se puede observar claramente que las 16 mezclas resultan importantes en el desempeño del motor, aunque se tomaron únicamente sus niveles mínimo y máximo sin puntos intermedios.

Es muy interesante el resultado para el Nitrometano $100 \%\left(\mathrm{CH}_{3} \mathrm{NO}_{2}\right)$ en sus niveles $5 \%$ y $15 \%$, lo que lleva a forzar el conocimiento químico en la interpretación, tal vez, de la característica de la rápida evaporación de este producto químico en el interior del motor, lo cual genera un enfriamiento en este, justificando el resultado obtenido de reducidas temperaturas.

La realización de investigaciones aplicadas por medio de la experimentación directa, si bien resultan costosas en 
recursos, se tiene que el valor aportado al investigador en la interpretación de efectos físicos, químicos y de la naturaleza misma, resulta de valor incalculable cuando investigaciones subsecuentes deban ser realizadas.

Los tratamientos estadísticos, por aparentemente complejos, son desestimados con altísima frecuencia por profesionales de diferentes disciplinas, pero con trabajos como este se podría impulsar a estos investigadores no doctos en la estadística a asumir la responsabilidad de su entendimiento y uso, y a socializar sus metodologías para beneficio de los grupos de interés.

\section{Referencias bibliográficas}

Argüelles, D.C. et al. (2013). Guía para la presentación de trabajos científicos bajo el estándar APA en la universidad EAN. Bogotá D. C.: Universidad EAN.

Gutiérrez Pulido, H., Salazar De la Vara, R. (2012). Análisis $y$ diseño de experimentos. México: McGraw-Hill / Interamericana Editores, S.A. de C.V.

Gutiérrez Pulido, H., Salazar De la Vara, R. (2009). Control estadístico de calidad y seis sigma. México: McGraw-Hill / Interamericana Editores, S.A. de C.V.

Kume, H. (2002). Herramientas estadísticas básicas para el mejoramiento de la calidad. Bogotá D. C.: Editorial Norma.

Montgomery, D. C., Runger, G. C. (1996). Probabilidad y estadística aplicadas a la ingeniería. México: McGraw-Hill. 
Al presente artículo se adjunta la guía de laboratorio, del Laboratorio de Ciencias Básicas de la Facultad de Ingeniería:

\begin{tabular}{|l|l|}
\hline \multicolumn{2}{|c|}{ IDENTIFICACIÓN } \\
\hline Programa académico: & Maestría en Ingeniería de Procesos. \\
\hline Unidad de estudio: & Control estadístico de procesos. \\
\hline Práctica 1: & Mezclas y preparación de soluciones. \\
\hline
\end{tabular}

